\title{
Measuring Emotional Wellbeing with a Non-intrusive Bed Sensor
}

\author{
Gert van der Vloed and Jelle Berentsen \\ Industrial Engineering \& Innovation Sciences \\ Eindhoven University of Technology, Den Dolech 2. 5612 AZ, Eindhoven \\ $\{$ G.v.d.Vloed, J.Berentsen\}@tue.nl
}

\begin{abstract}
We examine the possibility of using non-intrusive bed sensor measures to ascertain the emotional wellness of an individual. To this end we did a convergent validation study to determine whether heart rate and respiration measures provided by the bed sensor correlate with self reports through questionnaires. The results show that negative affect and worry tension positively correlate with nocturnal respiration.
\end{abstract}

Keywords: Emotions; Emotional Wellbeing; Physiological Measures.

\section{Introduction}

Emotions are a primary determinant of wellbeing. In the evolution of our species, reflexive and emotional responses to our environment played a vital role. On being confronted with a predator a human displays the emotion of fear and consequently the sympathetic nervous system triggers the rapid release of energy resources to initiate the fight/flight response. Without this sympathetic response, the possibility of adaptively coping with the such an emotional situation would radically decrease. Although these emotional reactions were evolutionarily selected for in a time very different from modern times they still function as a quick means of the human organism for evaluating its current condition and for selecting between possible behavioral responses [4].

When one gets positively or negatively excited, the sympathetic branch of the autonomic nervous system (ANS) gets activated. This sympathetic activation increases the readiness of the organism to come into action; it raises heart rate, increases respiration, raises blood pressure and decreases heart rate variability. Negative emotions such as anger and fear are associated with stronger increases in sympathetic activation than positive emotions such as happiness and contentment [2]. In addition to these stronger activations, the physiological consequences of negative emotions are longer lasting compared to positive emotions $[2,7]$. Furthermore, it has been shown [3] that daily stress, worry and rumination lead to an increased heart rate (HR) and a decreased heart rate variability (HRV). Detecting changes in physiological measures (such as HR, HRV, respiration and blood pressure) can thus be indications of changes in that person's emotional wellbeing (in addition to being indicators of other problems 
such as coronary heart disease). This raises the possibility of identifying changes in emotional states through measuring differences in ANS activity. These physiological changes should then be more indicative of negative emotions than positive emotions due to their greater intensity and temporal extent. There has been some interesting work in HCI research on the use of non-intrusive emotion detection through measuring physiology (e.g.[1, 6, 10]). Our research differs from these studies in that our focus lies on detecting long term changes in emotional wellbeing instead of detecting short term instantaneous effects of emotional states.

A predominance of negative emotional states leads to a decrement in emotional wellbeing and even physical wellbeing. There is a strong link between mental health and physical health. Numerous studies have shown that psychological stress can lead to decreased immune response (e.g. [5]). Stress also lowers the number of cytotoxic lymphocytes called "natural killer cells" that protect the body not only against infectious diseases but also play an important role in the rejection of tumors. Therefore, monitoring a person's wellbeing and responding to threats to this wellbeing (such as sustained negative emotions) should not only be effective in enhancing that person's emotional wellness but also enhance that person's physical wellbeing, care dependency and ultimately life expectancy.

The standard method for measuring changes in a person's emotional wellbeing is having them fill out questionnaires. In order to avoid this we investigate automated alternatives that measure physiological and/or behavioral changes that can be indicative of emotional change. The goal of our research is to provide non-intrusive automated measures of emotional wellbeing.

\section{Measuring Nocturnal Physiology Using a Bed Sensor}

Taking nocturnal measures of heart rate, respiration and activity provides a unique opportunity to gather data in a non-intrusive way over a long stretch of time. There are two additional advantages associated with the use of these physiological signals as a measure for emotional wellbeing. First, one would expect the general emotional state to be least contaminated by specific situational cues during sleep. The individual is essentially unresponsive to sensory stimulation (except perhaps the comfort of the bed, temperature and excessive noise). From the perspective of an individual's general emotional state this would thus provide an ideal measurement, because of the relative absence of sensory stimulation in the temporal proximity. The ANS activity would thus reflect a more general context independent measure of the individual's emotional state at that time. Relatedly, taking nocturnal measures increases the chance of distinguishing negative emotional states from positive emotional states because of their greater temporal extent.

As a means for taking physiological measures in a non-intrusive way we chose to investigate the use of a bed sensor. The bed sensor used in our research is the Emfit DVM (discrete vitals monitoring) sensor (as depicted in Figure 1) which is a thin film dynamic sensor, installed under the mattress. It responds to small pressure changes caused by a person's ballistocardiographic (BCG), respiration movements and other bodily movement during sleep. The output of the sensor is a single voltage signal corresponding with all pressure changes caused by the a person lying on the mattress. 


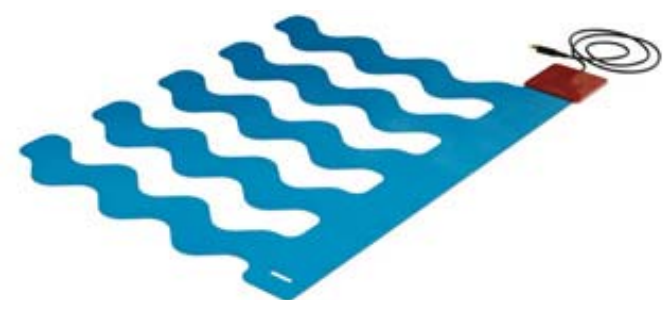

Fig. 1. Dicrete vitals monitoring sensor

The digital data acquisition and signal processing unit uses specialized algorithms to calculate heart and respiration rates and movement activity from the sensor signal. The sampling frequency of the sensor is $200 \mathrm{~Hz}$.

Because the sensor is thin and capable of taking recordings of the above mentioned physiological traits through the mattress it is minimally intrusive. The sensor causes no noticeable difference for a person lying in bed (apart perhaps from being conscious that the sensor is there independent of differences in sensation).

\section{Convergent Validation Study}

In order to evaluate bed sensor measures of physiology as a valid means for probing the emotional wellbeing of an individual, there is a need for alternate measures of emotional states. Self report measures such as questionnaires or interviews are still the most authoritative means for taking these measures despite shortcomings such as the possibility of using response strategies, conforming to social desirability and the more fundamental problem of whether emotional states are sufficiently accessible to an individual consciousness (suppressed emotions are per definition not consciously accessible). The questionnaires that were used in our research as convergent validation measures are the "Positive And Negative Affect Schedule" (PANAS) [11] and the "Affect Grid" [9]. Both these questionnaires assume a two dimensional model of affect. However, where the PANAS assumes independence between positive and negative affect dimensions, the Affect Grid puts these on opposite ends of the hedonism dimension and adds arousal as the second dimension. Furthermore, we investigated the effects of Stress and Worry by adapting questionnaires used in previous research [8].

Correlations were predicted between the Heart Rate and Respiration measures on the one side and the Negative Affect (PANAS), Hedonism, Arousal, Worry and Stress on the other. Higher negative affect, lower hedonism, higher arousal, higher worry and stress are all hypothesized to be predictive of increases in Heart Rate and Respiration and a decrease in Heart Rate Variability (HRV).

\section{Results and Conclusion}

The initial results over three subjects (measured 23 days each) show that of the expected correlations between physiological measures provided by the bed sensor and 
the questionnaire measures only the correlations between Negative Affect and Respiration $(r=0.41, p<0.001)$, between Stress and Respiration $(r=0.29, p<0.05)$ and between Worry tension and Respiration $(\mathrm{r}=0.27, \mathrm{p}<0.05)$ were significant. These correlations were calculated on the z-scores of the individual subjects as to compensate for individual differences in baseline physiological activity and style for filling out questionnaires. Nocturnal breathing frequency correlates positively with the reported negative affect, stress and tension associated with worry reported the night before.

At this point, we conclude that there are indications that physiological measures taken with a non-intrusive bed sensor correlate with emotional wellbeing; a prevalence of negative emotions, stress as well as tension associated with worry during the day, lead to significantly higher respiration rates during the night. This opens up possibilities for the automated non-intrusive measurement of emotional wellbeing.

Acknowledgement. This work was supported by the SenterNovem Point One project 'IPTV Portal to Wellness', part of the ITEA-2 project AmIE.

\section{References}

1. Anttonen, J., Surakka, V.: Emotions and heart rate while sitting on a chair. In: Proceedings of the SIGCHI Conference on Human Factors in Computing Systems, pp. 491-499. ACM, New York (2005)

2. Baumeister, R.F., Bratslavsky, E., Finkenauer, C., Vohs, K.D.: Bad is stronger than good. Rev. Gen. Psychol. 5, 323-370 (2001)

3. Brosschot, J.F., van Dijk, E., Thayer, J.F.: Daily worry is related to low heart rate variability during waking and the subsequent nocturnal sleep period. Int. J. Psychophysiol. 63, 3947 (2007)

4. Cosmides, L., Tooby, J.: Evolutionary psychology and the emotions. In: Lewis, M., Haviland-Jones, J.M. (eds.) Handbook of Emotions, 2nd edn., pp. 91-115. Guilford, NY (2000)

5. Glaser, R., Rice, J., Sheridan, J., Fertel, R., Stout, J.C.: Stress-related immune suppression: Health implications. Brain Behav. Immun. 1, 7-20 (1987)

6. Horlings, R., Datcu, D., Rothkrantz, L.J.: Emotion recognition using brain activity. In: Proceedings of the 9th international Conference on Computer Systems and Technologies and Workshop For PhD Students in Computing, vol. 374. ACM, New York (2008)

7. Pieper, S., Brosschot, J.F.: Prolonged stress-related cardiovascular activation: is there any? Ann. Behav. Med. 30, 91-103 (2005)

8. Pieper, S., Brosschot, J.F., van der Leeden, R., Thayer, J.F.: Cardiac effects of momentary assessed worry episodes and stressful events. Psychosom. Med. 69, 901-909 (2007)

9. Russell, J.A., Weiss, A., Mendelsohn, G.A.: Affect Grid: A single-item scale of pleasure and arousal. J. Pers. Soc. Psychol. 57, 493-502 (1989)

10. Wang, H., Prendinger, H., Igarashi, T.: Communicating emotions in online chat using physiological sensors and animated text. In: CHI 2004 Extended Abstracts on Human Factors in Computing Systems, pp. 1171-1174. ACM, New York (2004)

11. Watson, D., Clark, L.A., Tellegen, A.: Development and validation of brief measures of positive and negative affect:The PANAS scales. J. Pers. Soc. Psychol. 54, 1063-1070 (1998) 